\title{
Genetic Diversity of Fusarium oxysporum f. sp. cubense in East and Central Africa
}

\begin{abstract}
Patrick Karangwa, ${ }^{\dagger}$ Department of Plant Pathology, University of Stellenbosch, Matieland 7602, South Africa, and Rwanda Agriculture and Animal Resources Development Board, Kigali, Rwanda; Diane Mostert and Privat Ndayihanzamaso, Department of Plant Pathology, University of Stellenbosch, Matieland 7602, South Africa; Thomas Dubois, World Vegetable Center, Duluti, Arusha, Tanzania; Björn Niere, Julius Kühn-Institut, 38104 Braunschweig, Germany; Alexandra zum Felde, Organic Plant Production and Agroecosystems Research in the Tropics and Subtropics, Faculty of Organic Agricultural Sciences, University of Kassel, 37213 Witzenhausen, Germany; Alexander Schouten, Molecular Phytomedicine, Institute of Crop Science and Resource Conservation, Faculty of Agricultural Sciences, University of Bonn, 53115 Bonn, Germany; Guy Blomme, Bioversity International Addis Office, c/o ILRI, Addis Ababa, Ethiopia; Fenton Beed, World Vegetable Center, Kasetsart University, Bangkok, Thailand; and Altus Viljoen, ${ }^{\dagger}$ Department of Plant Pathology, University of Stellenbosch, Matieland 7602, South Africa
\end{abstract}

\begin{abstract}
Banana Fusarium wilt is a major production constraint globally and a significant threat to the livelihoods of millions of people in East and Central Africa (ECA). A proper understanding of the diversity and population dynamics of the causal agent, Fusarium oxysporum f. sp. cubense (Foc), could be useful for the development of sustainable disease management strategies for the pathogen. The current study investigated the diversity of Foc in ECA using vegetative compatibility group (VCG) analysis, PCR-RFLPs of the ribosomal DNA's intergenic spacer region, as well as phylogenetic analysis of the elongation factor- $1 \alpha$ gene. Six VCGs $(0124,0125,0128,01212,01220$, and 01222), which all belong to one lineage (Foc lineage VI), were widely distributed throughout the

region. VCGs 0128 and 01220 are reported for the first time in Burundi, the Democratic Republic of Congo (DRC), Rwanda, Tanzania, and Uganda, while VCG 01212 is reported in the DRC and Rwanda. Isolates that did not belong to any of the known VCGs were identified as Foc lineage VI members by phylogenetic analysis and may represent novel VCGs. CAV 2734, a banana pathogen collected in Rwanda, clustered with nonpathogenic $F$. oxysporum isolates in lineage VIII. Results from this study will contribute significantly toward the implementation of banana Fusarium wilt disease management practices in the region, such as the restricted movement of infected planting material and the selective planting of resistant banana varieties.
\end{abstract}

The fungus Fusarium oxysporum f. sp. cubense (E.F. Smith) W.C. Snyder \& H.N. Hansen (Foc) is the causal agent of Fusarium wilt, an important disease of banana and one of the most destructive of all plant diseases (Simmonds 1966; Stover 1962). In East and Central Africa (ECA), the disease is the major threat to the sustainability of banana processing and export initiatives, as these generally rely on cultivars that are highly susceptible to Foc race 1 (Karangwa et al. 2016). While many banana production constraints may be manageable, the management of Fusarium wilt is restricted by the paucity of effective and reliable control options (Ploetz 2015). The use of resistant cultivars is most effective, but the choice of cultivar depends on the character of the pathogen. An in-depth understanding of the genetic diversity of Foc, therefore, is crucial for development of effective and durable management strategies.

Initial studies on the diversity of Foc focused on variation in pathogenicity, and recognized three races with selective pathogenicity to certain banana cultivars (Stover and Buddenhagen 1986; Stover and Simmonds 1987; Waite and Stover 1960). However, the race structure in Foc is not clearly defined, and pathogenicity testing of $F$. oxysporum pathogens has been shown to be influenced by a number of variables including host age (Hart and Endo 1981) and method

${ }^{\dagger}$ Corresponding authors: Patrick Karangwa,

E-mail: patrick.karangwa1@gmail.com; and Altus Viljoen,

E-mail: altus@sun.ac.za

The Belgian Directorate General of Development Cooperation (DGDC) funded this work through the Consortium for Improving Agricultural Livelihoods in Central Africa (CIALCA), with Bioversity International as the lead CGIAR institution (grant LoA CfL 2009/4).

*The $\boldsymbol{e}$-Xtra logo stands for "electronic extra" and indicates that one supplementary figure is published online.

Accepted for publication 6 October 2017.

@ 2018 The American Phytopathological Society of inoculation (Kraft and Haglund 1978). Further investigations into Foc diversity beyond physiological races have focused on vegetative compatibility, the natural ability of filamentous fungi to merge their mycelium through the process of anastomosis, when they are genetically similar (Glass et al. 2000). So far, 24 vegetative compatibility groups (VCGs) have been identified within Foc (Fourie et al. 2011).

Interest in VCGs as genetic markers stems from the assumption that isolates sharing common alleles at all vic loci would be genetically very similar for the rest of their genomes (Glass et al. 2000; Leslie and Summerell 2006). Though useful as an identification tool for genetically related genotypes, vegetative compatibility does not inform on the degree of relatedness between vegetatively compatible strains or on genetic distance between vegetatively incompatible strains. A mutation in even a single vic locus would place two otherwise identical isolates in different VCGs (Glass et al. 2000; Leslie and Summerell 2006).

Studies of Foc's genetic diversity and evolutionary relationships have advanced considerably over the past two decades. They now involve molecular and phylogenetic methods that have enabled the discovery of Foc lineages that constitute a meaningful classification of genetic variation within the pathogen (Bentley et al. 1998; Fourie et al. 2009, 2011; Groenewald et al. 2006; Koenig et al. 1997; O’Donnell et al. 1998).

Plant pathogen diversity and evolutionary studies are presumably more informative in the host's centers of origin and diversification than elsewhere. Having nurtured the evolution of the Foc race 1-resistant East African highland bananas (EAHB; AAA genome), ECA is considered the banana's secondary center of diversity after Southeast Asia (Stover and Simmonds 1987). This region also harbors two Foc VCGs that have not yet been reported anywhere else in the world, i.e., VCGs 01212 and 01214, and one VCG so far reported only in eastern Africa and Southeast Asia (VCG 01222) (Bentley et al. 2001; Ploetz and Pegg 2000). VCG 01214 has been reported from an isolated area located near Malawi's border with Tanzania (Ploetz et al. 1990), and was found to be evolutionarily distant to other Foc VCGs (Bentley et al. 1998; Fourie et al. 2009; Koenig et al. 1997).

Previous surveys conducted in ECA (Burundi, DRC, Kenya, Rwanda, Uganda, and Tanzania) revealed that VCGs 0124 and 0125 are ubiquitously found in the region. VCGs 0128 and 01220 , however, 
were only found in Kenya, VCG 01212 in Kenya, Tanzania, and Uganda, and VCG 01222 in Kenya and Uganda (Kangire et al. 2001; Kung'u and Jeffries 1998; Ploetz et al. 1990; Rutherford 2001). VCGs 0124, 0125, 0128, 01212, 01220, and 01222 are all members of Foc lineage VI, and complexes among these VCGs are common. VCG 01214 is hitherto the only known member of Foc lineage VIII (Fourie et al. 2009). A "tropical" form of Foc race 4 (Foc TR4), previously confined to countries in Australasia, has recently been found in Africa when the fungus was discovered in Mozambique (Butler 2013). Foc TR4 includes strains belonging to the VCG 01213-01216 complex, which are members of Foc lineage V. Foc TR4 is particularly severe and affects a range of banana varieties well beyond the host range of Foc race 1 (Fourie et al. 2009).

The objective of the current study was to investigate Foc diversity in five ECA countries (Burundi, DRC, Rwanda, Uganda, and Tanzania). These countries are considered among the most important bananaproducing and -consuming countries in the world (Karamura et al. 1999; Lynam 2000; Rutherford 2001; http://faostat3.fao.org). Knowledge of Foc diversity in ECA could provide a basis to further investigate disease management options, such as the development of markers for the early and accurate detection of Foc in the region.

\section{Materials and Methods}

Sample collection, purification, and maintenance of isolates. Surveys for banana Fusarium wilt were conducted in 2009 (June, November, and December) and 2010 (May, June, and July), during which 442 plant samples were collected from plants showing typical Fusarium wilt symptoms. Externally, these plants displayed chlorosis and collapse of the oldest leaves, and internally (inside the pseudostem), a brown to brick red discoloration of the vascular system (Ploetz 2015). Collected samples included 168 samples from Rwanda, 100 from Tanzania, 100 from Burundi, and 74 samples from the DRC. Samples were also received from collaborators in the DRC (38 samples), Burundi (10 samples), and Uganda (85 samples). The sampling was extensive in Rwanda, Burundi, and Uganda, while it was limited to the Kagera and Kigoma regions in Tanzania and to the South and North Kivu provinces in the DRC due to logistical limitations. Samples were collected from all susceptible host cultivars grown in the region (Pisang Awak, Sukari Ndiizi, Gros Michel, Ney Poovan, and Bluggoe).

Fungal isolation was carried out at the Department of Plant Pathology, Stellenbosch University (USPP) in South Africa. For each sample, a segment of about $5 \mathrm{~mm}$ was cut from a dry pseudostem strand and placed in a Petri dish onto potato dextrose agar (PDA) that was supplemented with streptomycin (4 mg/liter). Cultures were incubated at $25^{\circ} \mathrm{C}$ for 7 days, after which they were purified and singlespored. A total of 281 pure cultures were tentatively identified as $F$. oxysporum based on cultural characteristics that included fluffy, cottony mycelia and the production of a pale to dark violet or dark magenta pigment in agar (Leslie and Summerell 2006). Two isolates of F. sacchari (E.J. Butler) W. Gams (CAV 2804 and 2837) and two isolates of F. semitectum Berk. \& Ravenel (CAV 2580 and CAV 2678) were also tentatively identified. All isolates are maintained on carnation leaf agar medium slants (Leslie and Summerell 2006) at $4^{\circ} \mathrm{C}$ as well as in $15 \%$ glycerol at $-80^{\circ} \mathrm{C}$ in the CAV culture collection of USPP.

DNA isolation. DNA was extracted from pure cultures grown on PDA at $25^{\circ} \mathrm{C}$ for 7 days using the Wizard SV Genomic DNA Purification Systems DNA extraction kit (Promega, Madison, U.S.A.). The manufacturer's instructions were followed, with a slight modification for the initial step. In this step, the mycelia were first vigorously shaken with glass beads (diameter: $2 \mathrm{~mm}$; Lasec, Cape Town, South Africa) in $400 \mu \mathrm{l}$ of the lysis buffer (provided with the kit) using a MM301 shaker (Retsch, Haan, Germany) set at $30 \mathrm{~Hz}$ for $5 \mathrm{~min}$, and then incubated for $10 \mathrm{~min}$ in a water bath at $65^{\circ} \mathrm{C}$. The lysate was thereafter centrifuged for $4 \mathrm{~min}$ at $4,000 \mathrm{rpm}$, and the liquid phase pipetted into the kit's columns. The extraction process then proceeded as described in the manufacturer's instructions.

IGS PCR-RFLP. Characterization of $F$. oxysporum isolates by PCR-RFLP analysis was conducted according to the technique developed by Fourie et al. (2009), which separates Foc into two clades (A/B) and eight lineages (I to VIII). For each isolate, the intergenic spacer (IGS) region was selectively amplified with primers PNFo and PnF22 (Edel et al. 1995) using a GeneAmp PCR System 9700 (Applied Biosystems, Foster City, U.S.A.). Each amplification reaction contained $\sim 5 \mathrm{ng} / \mu \mathrm{l}$ DNA, $0.3 \mu \mathrm{M}$ of each primer, $250 \mu \mathrm{M}$ dNTPs (Fermantas, Nunningen, Switzerland), $0.04 \mathrm{U} / \mu \mathrm{l}$ Taq DNA polymerase (Roche Molecular Biochemicals, Manheim, Germany), and PCR buffer with $\mathrm{MgCl}_{2}$ (Roche). PCR cycling conditions consisted of 35 cycles at $94^{\circ} \mathrm{C}$ for $45 \mathrm{~s}$, $50^{\circ} \mathrm{C}$ for $45 \mathrm{~s}$, and $72^{\circ} \mathrm{C}$ for $90 \mathrm{~s}$. Each PCR reaction was preceded by an initial denaturation step at $94^{\circ} \mathrm{C}$ for 2 min and concluded by a final extension step at $72^{\circ} \mathrm{C}$ for $5 \mathrm{~min}$. The RFLP reactions were conducted by subjecting all IGS amplicons to digestion with the restriction enzyme Eco88I (Aval) (Thermo Fischer Scientific, Massachusetts, U.S.A.), following the manufacturer's instructions, in order to differentiate Foc isolates into clades A and B. Clade A isolates were further digested with the BceAI enzyme, while clade B isolates were digested with BbvI (Fermantas, Nunningen, Switzerland) for identification of lineages. Isolates representative of each clade and lineage (Fourie et al. 2009) were used as reference isolates. All restriction products were separated by gel electrophoresis and visualized under UV light with a gel documentation and analysis unit (Syngene, Cambridge, U.K.).

Vegetative compatibility testing. Vegetative compatibility testing was conducted on 281 single spore isolates of $F$. oxysporum (Table 1), using nitrate non-utilizing (nit) mutants as described by Leslie and Summerell (2006). The nit mutants were generated on minimal medium and/or PDA supplemented with 1.5 to $4 \%$ potassium chlorate, and phenotyped as nit 1 , nit 3 , or Nit $\mathrm{M}$ mutants based on their inability to assimilate nitrogenous compounds (nitrate, nitrite, and hypoxanthine, respectively) (Leslie and Summerell 2006). For each isolate, nit 1 mutants were paired with the Nit $\mathrm{M}$ mutant of the same isolate to confirm heterokaryon self-compatibility, and with the NitM of tester isolates to determine VCG identity (Ploetz and Correll 1988). At least two mutants were generated per isolate and VCG testing was conducted at least twice for the same isolate to confirm vegetative compatibility. Heterokaryon compatibility was confirmed by visual observation as described by Leslie and Summerell (2006).

Sequencing and phylogenetic analysis. Thirteen $F$. oxysporum isolates that did not pair with the Foc VCG tester set, as well as the $F$. sacchari isolates and $F$. semitectum isolates, were selected for phylogenetic analysis. For each isolate, the translation elongation factor $1 \alpha$ (TEF) DNA region was amplified with the primer set EF1 and EF2 (O'Donnell et al. 1998) in a GeneAmp PCR System 9700 (Applied Biosystems). The PCR products were then purified using a High Pure PCR Product purification kit (Roche Applied Biochemicals), and sequenced in both directions with the original PCR primers using the 3130XL Genetic Analyzer (Applied Biosystems). TEF DNA sequences for the 17 isolates were phylogenetically compared with TEF sequences of 48 Foc isolates previously studied by Fourie et al. (2009). The TEF sequence of $F$. circinatum Nirenberg \& O'Donnell isolate FCC4880 (gb|KC514062) was included as outgroup.

Sequences were edited with Geneious bioinformatics software (Biomatters, Auckland, New Zealand) by trimming off low quality extremities and by generating consensus assemblies between the forward and reverse primer sequences, and then exported to MAFFT version 5.85 for multiple sequence alignment with the L-INS-I option effective (Katoh et al. 2005). All ambiguously aligned sites were excluded from further analyses. Phylogeny based on maximum likelihood (ML) was inferred for the TEF dataset using PhyML, version 3 (Guindon et al. 2010). The general time reversible (GTR) model (Tavare 1986) plus I were used, as indicated by Smart Model selection tool in PhyML (Lefort et al. 2017). Initial trees for the heuristic search were obtained automatically by applying neighbor-join and BioNJ algorithms to a matrix of pairwise distances estimated using the maximum composite likelihood (MCL) approach, and then selecting the topology with superior log likelihood value ( -1880.65590$)$. ML bootstrap confidence values were based on 1,000 replications. Trees were visualized and edited in Treegrah 2 version 2.14.0-771 (Stover and Müller 2010).

Pathogenicity testing. Isolates that did not pair with any of the known VCGs were subjected to pathogenicity testing, except those that were identified as $F$. semitecum (CAV 2580 and CAV 2678) and F. sacchari (CAV 2804 and CAV 2837). Pathogenicity testing 
was conducted with 4-month-old tissue culture-derived Gros Michel plantlets by using the method described by Dita et al. (2011). Three plants were inoculated per isolate and three positive controls were included in the experiment. After inoculation, the plantlets were kept at $25^{\circ} \mathrm{C}$ under a $16 / 8$-h photoperiod regime. Disease development was assessed 6 weeks after inoculation by cutting the rhizomes to examine vascular discoloration typical of Foc internal symptoms (Dita et al. 2011). The pathogen was reisolated from discolored rhizome tissue and transferred onto PDA to complete Koch's postulates.

\section{Results}

IGS PCR-RFLP. Restriction pattern profiles of IGS amplicons digested with the Eco88I (Aval) restriction enzyme divided ECA isolates into clades $\mathrm{A}$ and $\mathrm{B}$, with four isolates belonging to clade A while 274 isolates belonged to clade B (Table 1, Fig. 1). IGS amplicons of the four clade A isolates (CAV 2581, CAV 2633, CAV 2678, and CAV 2723), when digested with BceAI, resulted in restriction patterns that did not match any of the typical IGS profiles of Foc lineages I, II, III, IV, and V described by Fourie et al. (2009). Digestion of IGS amplicons of clade B isolates with BbvI restriction enzyme revealed that, among 274 clade B isolates, 268 belonged to Foc lineage VI (Fig. 2). All isolates that could not be separated into known Foc lineages as described by Fourie et al. (2009) (Table 1) were subjected to pathogenicity testing as well as phylogenetic analysis with reference Foc isolates to investigate their relationships with Foc.

Table 1. Clades, lineages, vegetative compatibility groups (VCGs), and origin of Fusarium oxysporum f. sp. cubense (Foc) characterized in the current study

\begin{tabular}{|c|c|c|c|c|c|c|c|c|c|}
\hline Isolate ID no. & Clade & Lineage & VCG & Origin & Isolate ID no. & Clade & Lineage & VCG & Origin \\
\hline CAV 2572 & B & Foc VI & 0124 & Tanzania & CAV 2644 & B & Foc VI & $0124 / 22$ & Tanzania \\
\hline CAV 2573 & B & Foc VI & 01222 & Tanzania & CAV 2646 & B & Foc VI & 01222 & Tanzania \\
\hline CAV 2574 & B & Foc VI & 01222 & Tanzania & CAV 2647 & B & Foc VI & $0124 / 22$ & Tanzania \\
\hline CAV 2575 & B & Foc VI & $0124 / 22$ & Tanzania & CAV 2648 & B & Foc VI & $0124 / 22$ & Tanzania \\
\hline CAV 2576 & $\mathrm{nt}^{\mathrm{a}}$ & Foc VI (phylo) ${ }^{\mathrm{b}}$ & incomp..$^{\mathrm{c}}$ & Tanzania & CAV 2649 & B & Foc VI & 01212 & Tanzania \\
\hline CAV 2577 & $\mathrm{~B}$ & Foc VI & $0125 / 8 / 20 / 22$ & Tanzania & CAV 2678 & A & A.P. ${ }^{d}$ & $\mathrm{nt}$ & Rwanda \\
\hline CAV 2578 & B & Foc VI & $0124 / 22$ & Tanzania & CAV 2745 & B & Foc VI & $0124 / 22$ & Burundi \\
\hline CAV 2579 & B & Foc VI & 0128 & Tanzania & CAV 2746 & B & Foc VI & 0124 & Burundi \\
\hline CAV 2580 & $\mathrm{nt}$ & $\mathrm{nt}$ & $\mathrm{nt}$ & Tanzania & CAV 2748 & B & Foc VI & $0124 / 22$ & Burundi \\
\hline CAV 2581 & $\mathrm{~A}$ & A.P. & incomp. & Tanzania & CAV 2749 & B & Foc VI & $0124 / 22$ & Burundi \\
\hline CAV 2582 & $\mathrm{~B}$ & Foc VI & 0124 & Tanzania & CAV 2750 & B & Foc VI (phylo) & incomp. & Burundi \\
\hline CAV 2583 & B & Foc VI & $0125 / 8 / 20 / 22$ & Tanzania & CAV 2752 & B & Foc VI & $0124 / 22$ & Burundi \\
\hline CAV 2586 & B & Foc VI & $0124 / 5 / 20 / 22$ & Tanzania & CAV 2753 & B & Foc VI & $0124 / 22$ & Burundi \\
\hline CAV 2587 & B & Foc VI & $0124 / 22$ & Tanzania & CAV 2755 & B & Foc VI & $0128 / 20$ & Burundi \\
\hline CAV 2588 & B & Foc VI & 0124 & Tanzania & CAV 2756 & B & Foc VI & $0128 / 20$ & Burundi \\
\hline CAV 2589 & B & Foc VI & 0128 & Tanzania & CAV 2759 & B & Foc VI & $0124 / 22$ & Burundi \\
\hline CAV 2590 & B & Foc VI & 0124 & Tanzania & CAV 2760 & B & Foc VI & $0124 / 22$ & Burundi \\
\hline CAV 2591 & B & Foc VI & $0124 / 22$ & Tanzania & CAV 2762 & B & Foc VI (phylo) & incomp. & Burundi \\
\hline CAV 2592 & B & Foc VI & 0125 & Tanzania & CAV 2763 & B & Foc VI & $0124 / 22$ & Burundi \\
\hline CAV 2593 & B & Foc VI & 01222 & Tanzania & CAV 2765 & B & Foc VI & $0124 / 8$ & Burundi \\
\hline CAV 2594 & B & Foc VI & 0125 & Tanzania & CAV 2766 & B & Foc VI & 01222 & Burundi \\
\hline CAV 2595 & nt & Foc VI (phylo) & incomp. & Tanzania & CAV 2770 & B & Foc VI & $0124 / 5 / 8 / 22$ & Burundi \\
\hline CAV 2596 & $\mathrm{~B}$ & Foc VI & 0128 & Tanzania & CAV 2773 & B & Foc VI & 01222 & Burundi \\
\hline CAV 2597 & $\mathrm{~B}$ & Foc VI & 0125 & Tanzania & CAV 2775 & B & Foc VI & $0124 / 22$ & Burundi \\
\hline CAV 2599 & $\mathrm{nt}$ & Foc VI (phylo) & incomp. & Tanzania & CAV 2776 & B & Foc VI & 0124 & Burundi \\
\hline CAV 2600 & $\mathrm{~B}$ & Foc VI & 01222 & Tanzania & CAV 2777 & B & Foc VI & $0124 / 22$ & Burundi \\
\hline CAV 2601 & B & Foc VI & 01212 & Tanzania & CAV 2778 & B & Foc VI & 0125 & Burundi \\
\hline CAV 2602 & B & Foc VI & $0124 / 22$ & Tanzania & CAV 2783 & B & Foc VI & 01220 & Burundi \\
\hline CAV 2604 & B & Foc VI & $0124 / 8 / 22$ & Tanzania & CAV 2784 & B & Foc VI & $0124 / 22$ & Burundi \\
\hline CAV 2605 & B & Foc VI & 0124 & Tanzania & CAV 2878 & B & Foc VI & $0124 / 22$ & Burundi \\
\hline CAV 2607 & B & Foc VI & $0124 / 22$ & Tanzania & CAV 2787(a) & B & Foc VI & 01222 & Burundi \\
\hline CAV 2608 & B & Foc VI & 0124 & Tanzania & CAV 2788 & B & Foc VI & $0124 / 22$ & Burundi \\
\hline CAV 2609 & B & Foc VI & $0124 / 22$ & Tanzania & CAV 2790 & B & Foc VI & 0125 & Burundi \\
\hline CAV 2611 & B & $\mathrm{nt}$ & incomp. & Tanzania & CAV 2793 & B & Foc VI & $0124 / 22$ & Burundi \\
\hline CAV 2612 & B & Foc VI & 0124 & Tanzania & CAV 2794 & B & Foc VI & 0128 & Burundi \\
\hline CAV 2614 & B & Foc VI & 0124 & Tanzania & CAV 2795 & B & Foc VI & $0124 / 22$ & Burundi \\
\hline CAV 2615 & B & Foc VI & 01222 & Tanzania & CAV 2796 & B & Foc VI & 0124 & Burundi \\
\hline CAV 2616 & B & Foc VI & 01212 & Tanzania & CAV 2797 & $\mathrm{~B}$ & Foc VI & $0125 / 8 / 20 / 22$ & Burundi \\
\hline CAV 2617 & B & Foc VI & 0128 & Tanzania & CAV 2798 & B & Foc VI & $0124 / 22$ & Burundi \\
\hline CAV 2618 & B & Foc VI & 01212 & Tanzania & CAV 2799 & B & Foc VI & $0124 / 22$ & Burundi \\
\hline CAV 2619 & B & Foc VI & $0124 / 22$ & Tanzania & CAV 2800 & B & Foc VI & 0124 & Burundi \\
\hline CAV 2620 & B & Foc VI & $0125 / 8$ & Tanzania & CAV 2804 & nt & $\mathrm{nt}$ & $\mathrm{nt}$ & Burundi \\
\hline CAV 2621 & B & Foc VI & 01222 & Tanzania & CAV 2805 & B & Foc VI & $0124 / 22$ & Burundi \\
\hline CAV 2624 & B & Foc VI & 0128 & Tanzania & CAV 2806 & B & Foc VI & $0124 / 22$ & Burundi \\
\hline CAV 2625 & B & Foc VI & 01222 & Tanzania & CAV 2807 & B & Foc VI & $0124 / 22$ & Burundi \\
\hline CAV 2628 & B & Foc VI & $0124 / 22$ & Tanzania & CAV 2809 & B & Foc VI & 0124 & Burundi \\
\hline CAV 2630 & B & Foc VI & 0124 & Tanzania & CAV 2810 & B & Foc VI & 01222 & Burundi \\
\hline CAV 2632 & B & Foc VI & 01212 & Tanzania & CAV 2813 & B & Foc VI & 01222 & Burundi \\
\hline & & & & & & & & \multicolumn{2}{|c|}{ (Continued on next page) } \\
\hline
\end{tabular}

${ }^{a}$ nt: Not tested.

${ }^{\mathrm{b}}$ Foc VI (phylo): Isolate found associated with Foc lineage VI by phylogenetic analysis. All other Foc VI isolates were identified by IGS-PCR-RFLP.

c incomp.: Isolate found incompatible with all 24 known Foc VCGs.

d A.P.: Atypical profile, i.e., none of the profiles described by Fourie et al. (2009). 
Vegetative compatibility group analysis. Six VCGs of Foc (0124, $0125,0128,01212,01220$, and 01222) were identified in ECA (Table 1). Four of these, VCGs 0124, 0125, 0128, and 02222, were found in relatively similar frequencies in all five countries where samples were collected. VCG 01212 was absent in Burundi, and VCG 01220 was the least represented among the VCGs, with only one or two isolates per country (Table 1). VCGs 0124, 01222, and the VCG complex 0124-01222 (i.e., isolates belonging to both VCGs) were found to predominate in the region, with $70 \%$ of all Foc isolates (194 isolates) belonging to VCGs 0124, 01222, and the VCG complex 0124/22. Other VCG complexes found included 0124/5, 0124/8,
0124/20, 0125/8, 0125/22, 0128/20, 0124/5/22, 0124/5/8/22, and 0124/5/8/20 (Table 1). No complexes were observed between VCG 01212 and any of the other VCGs. Thirteen $F$. oxysporum isolates did not pair with any of the reference VCG Foc testers and of these, four were later confirmed to be nonpathogens (Table 2). Eight of the remaining nine isolates were self-compatible, but CAV 2750 was self-incompatible. CAV 2576 paired with CAV 2762 and CAV 2858, and CAV 2595 paired with CAV 2640. Isolates CAV 2599, CAV 2880, and CAV 2734 were not compatible to any other isolates.

Sequencing and phylogenetic analysis. The TEF data set tree's topology, evaluated with 1,000 likelihood bootstrap replications,

Table 1. (Continued from previous page)

\begin{tabular}{|c|c|c|c|c|c|c|c|c|c|}
\hline Isolate ID no. & Clade & Lineage & VCG & Origin & Isolate ID no. & Clade & Lineage & VCG & Origin \\
\hline CAV 2633 & A & A.P. & incomp. & Tanzania & CAV 2814 & B & Foc VI & 01222 & Burundi \\
\hline CAV 2634 & B & Foc VI & $0124 / 22$ & Tanzania & CAV 2815 & B & Foc VI & $0124 / 22$ & Burundi \\
\hline CAV 2635 & B & Foc VI & 01220 & Tanzania & CAV 2816 & B & Foc VI & 0124 & Burundi \\
\hline CAV 2637 & B & Foc VI & $0128 / 20$ & Tanzania & CAV 2817 & B & Foc VI & 0124 & Burundi \\
\hline CAV 2638 & $\mathrm{~B}$ & Foc VI & 0125 & Tanzania & CAV 2818 & $\mathrm{~B}$ & Foc VI & 01222 & Burundi \\
\hline CAV 2639 & $\mathrm{~B}$ & Foc VI & 01212 & Tanzania & CAV 2819 & B & Foc VI & 01222 & Burundi \\
\hline CAV 2640 & B & Foc VI (phylo) & incomp. & Tanzania & CAV 2820 & B & Foc VI & 01222 & Burundi \\
\hline CAV 2641 & $\mathrm{~B}$ & Foc VI & 01212 & Tanzania & CAV 2821 & B & Foc VI & 01222 & Burundi \\
\hline CAV 2643 & $\mathrm{~B}$ & Foc VI & $0124 / 22$ & Tanzania & CAV 2823 & B & Foc VI & $0124 / 22$ & Burundi \\
\hline CAV 2824 & B & Foc VI & 0124 & Burundi & CAV 2727 & B & Foc VI & 0124 & Rwanda \\
\hline CAV 2650 & $\mathrm{~B}$ & Foc VI & $0124 / 22$ & Rwanda & CAV 2728 & B & Foc VI & $0124 / 22$ & Rwanda \\
\hline CAV 2651 & $\mathrm{~B}$ & Foc VI & $0124 / 22$ & Rwanda & CAV 2729 & B & Foc VI & $0124 / 22$ & Rwanda \\
\hline CAV 2652 & B & Foc VI & 0124 & Rwanda & CAV 2730 & B & Foc VI & 0124 & Rwanda \\
\hline CAV 2653 & B & Foc VI & 0125 & Rwanda & CAV 2732 & B & Foc VI & $0124 / 5$ & Rwanda \\
\hline CAV 2654 & B & Foc VI & $0124 / 22$ & Rwanda & CAV 2733 & B & Foc VI & 0125 & Rwanda \\
\hline CAV 2655 & B & Foc VI & $0124 / 22$ & Rwanda & CAV 2734 & B & $\mathrm{nt}$ & incomp. & Rwanda \\
\hline CAV 2656 & B & Foc VI & $0124 / 22$ & Rwanda & CAV 2735 & B & Foc VI & 0124 & Rwanda \\
\hline CAV 2657 & B & Foc VI & $0124 / 22$ & Rwanda & CAV 2736 & B & Foc VI & $0124 / 22$ & Rwanda \\
\hline CAV 2658 & B & Foc VI & 0124 & Rwanda & CAV 2737 & B & Foc VI & $0124 / 22$ & Rwanda \\
\hline CAV 2660 & B & Foc VI & $0124 / 22$ & Rwanda & CAV 2738 & B & Foc VI & 0128 & Rwanda \\
\hline CAV 2661 & B & Foc VI & $0124 / 22$ & Rwanda & CAV 2740 & B & Foc VI & 0124 & Rwanda \\
\hline CAV 2662 & B & Foc VI & $0124 / 22$ & Rwanda & CAV 2741 & B & Foc VI & 0128 & Rwanda \\
\hline CAV 2663 & B & Foc VI & 01222 & Rwanda & CAV 2742 & B & Foc VI & $0124 / 22$ & Rwanda \\
\hline CAV 2665 & B & Foc VI & 0124 & Rwanda & CAV 2743 & B & Foc VI & $0124 / 22$ & Rwanda \\
\hline CAV 2666 & B & Foc VI & $0124 / 22$ & Rwanda & CAV 2744 & B & Foc VI & $0124 / 22$ & Rwanda \\
\hline CAV 2667 & B & Foc VI & $0124 / 22$ & Rwanda & CAV 2825 & B & Foc VI & $0125 / 8$ & DRC \\
\hline CAV 2669 & B & Foc VI & $0124 / 22$ & Rwanda & CAV 2826 & B & Foc VI & $0125 / 8$ & DRC \\
\hline CAV 2670 & B & Foc VI & $0124 / 22$ & Rwanda & CAV 2828 & B & Foc VI & 01222 & DRC \\
\hline CAV 2671 & B & Foc VI & $0124 / 22$ & Rwanda & CAV 2829 & B & Foc VI & 01222 & DRC \\
\hline CAV 2672 & B & Foc VI & 0124 & Rwanda & CAV 2830 & B & Foc VI & 0125 & $\mathrm{DRC}$ \\
\hline CAV 2673 & B & Foc VI & $0124 / 22$ & Rwanda & CAV 2831 & B & Foc VI & $0124 / 5 / 20 / 22$ & $\mathrm{DRC}$ \\
\hline CAV 2674 & B & Foc VI & 0124 & Rwanda & CAV 2833 & B & Foc VI & $0124 / 22$ & DRC \\
\hline CAV 2675 & B & Foc VI & 0124 & Rwanda & CAV 2834 & B & Foc VI & 01222 & DRC \\
\hline CAV 2676 & B & Foc VI & 01222 & Rwanda & CAV 2835 & B & Foc VI & $0124 / 22$ & DRC \\
\hline CAV 2677 & B & Foc VI & $0124 / 22$ & Rwanda & CAV 2836 & B & Foc VI & 01222 & DRC \\
\hline CAV 2679 & B & Foc VI & 0124 & Rwanda & CAV 2837 & $\mathrm{nt}$ & nt & nt & DRC \\
\hline CAV 2680 & B & Foc VI & $0124 / 22$ & Rwanda & CAV 2838 & B & Foc VI & $0124 / 5 / 22$ & DRC \\
\hline CAV 2681 & B & Foc VI & 01222 & Rwanda & CAV 2839 & B & Foc VI & 01222 & DRC \\
\hline CAV 2682 & B & Foc VI & 01222 & Rwanda & CAV 2840 & B & Foc VI & 0124 & DRC \\
\hline CAV 2683 & $\mathrm{~B}$ & Foc VI & 01212 & Rwanda & CAV 2841 & B & Foc VI & 0124 & DRC \\
\hline CAV 2684 & $\mathrm{~B}$ & Foc VI & $0124 / 22$ & Rwanda & CAV 2842 & $\mathrm{~B}$ & Foc VI & $0124 / 22$ & DRC \\
\hline CAV 2686 & B & Foc VI & 0124 & Rwanda & CAV 2843 & $\mathrm{~B}$ & Foc VI & 01222 & $\mathrm{DRC}$ \\
\hline CAV 2687 & B & Foc VI & $0124 / 22$ & Rwanda & CAV 2845 & $\mathrm{~B}$ & Foc VI & 01222 & $\mathrm{DRC}$ \\
\hline CAV 2688 & B & Foc VI & 01222 & Rwanda & CAV 2846 & B & Foc VI & $0124 / 22$ & DRC \\
\hline CAV 2689 & B & Foc VI & 01220 & Rwanda & CAV 2848 & $\mathrm{~B}$ & Foc VI & $0124 / 22$ & $\mathrm{DRC}$ \\
\hline CAV 2690 & B & Foc VI & 0124 & Rwanda & CAV 2849 & B & Foc VI & 01222 & DRC \\
\hline CAV 2693 & $\mathrm{~B}$ & Foc VI & 0128 & Rwanda & CAV 2850 & $\mathrm{~B}$ & Foc VI & $0128 / 20$ & DRC \\
\hline CAV 2695 & B & Foc VI & $0124 / 5 / 8 / 22$ & Rwanda & CAV 2851 & $\mathrm{~B}$ & Foc VI & $0124 / 22$ & DRC \\
\hline CAV 2696 & B & Foc VI & 0124 & Rwanda & CAV 2852 & B & Foc VI & $0125 / 8 / 20$ & DRC \\
\hline CAV 2699 & $\mathrm{~B}$ & Foc VI & 01220 & Rwanda & CAV 2853 & $\mathrm{~B}$ & Foc VI & $0124 / 22$ & DRC \\
\hline CAV 2700 & B & Foc VI & 0124 & Rwanda & CAV 2854 & B & Foc VI & $0124 / 22$ & DRC \\
\hline CAV 2702 & B & Foc VI & $0124 / 5 / 22$ & Rwanda & CAV 2855 & $\mathrm{~B}$ & Foc VI & 0128 & DRC \\
\hline CAV 2703 & $\mathrm{~B}$ & Foc VI & $0125 / 22$ & Rwanda & CAV 2856 & B & Foc VI & $0124 / 22$ & DRC \\
\hline CAV 2706 & B & Foc VI & $0124 / 22$ & Rwanda & CAV 2858 & B & Foc VI (phylo) & incomp. & $\mathrm{DRC}$ \\
\hline & & & & & & & & \multicolumn{2}{|c|}{ (Continued on next page) } \\
\hline
\end{tabular}


was well supported with high bootstrap values at nodes that separate the monophyletic ingroup from the outgroup $(99 \%)$, clade A $(83 \%)$ from clade B (88\%), as well as lineage VI from other clade B lineages (93\%). Of the 17 isolates analyzed, eight clustered within clade B's lineage VI, while none of the isolates was a member of clade A (Fig. 3). Four isolates, including one from Tanzania (CAV 2580), one from Rwanda (CAV 2678), one from DRC (CAV 2837), and one from Burundi (CAV 2804) grouped basal to the clades A and B with bootstrap

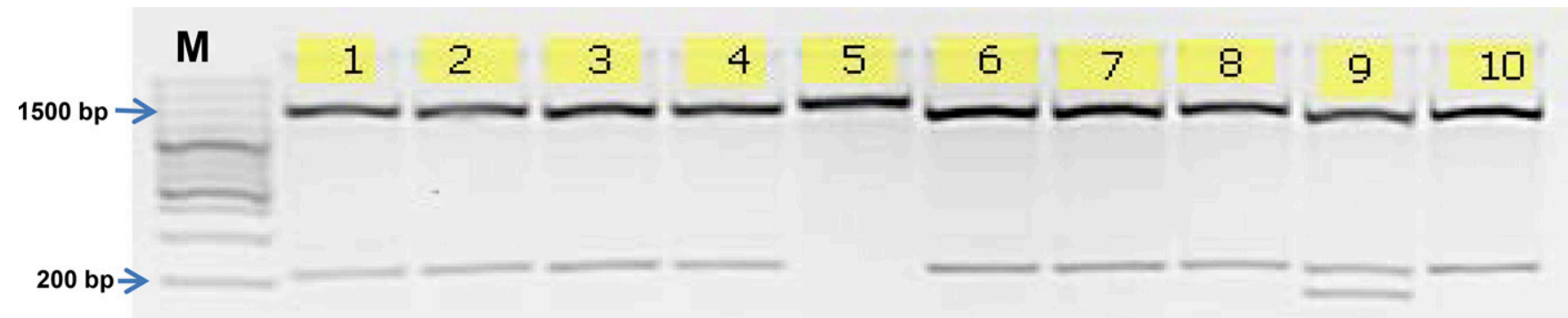

Fig. 1. Electrophoretic PCR-RFLP profiles of Eco88l-digested Fusarium oxysporum f. sp. cubense isolates from Tanzania. $M=$ molecular weight marker. Lanes 1-4, 6-8, and 10 represent clade B, lane 9 represents clade A, and lane 5 represents an atypical pattern as it exhibits neither clade A nor clade B pattern around 200 bp (absent pattern) but shares with both clades $A$ and $B$ the common large fragment of about 1,500 bp.

Table 1. (Continued from previous page)

\begin{tabular}{|c|c|c|c|c|c|c|c|c|c|}
\hline Isolate ID no. & Clade & Lineage & VCG & Origin & Isolate ID no. & Clade & Lineage & VCG & Origin \\
\hline CAV 2707 & B & Foc VI & $0124 / 22$ & Rwanda & CAV 2859 & B & Foc VI & $0124 / 22$ & DRC \\
\hline CAV 2708 & B & Foc VI & $0124 / 22$ & Rwanda & CAV 2860 & B & Foc VI & $0124 / 22$ & DRC \\
\hline CAV 2711 & B & Foc VI & $0124 / 22$ & Rwanda & CAV 2861 & B & Foc VI & $0124 / 22$ & DRC \\
\hline CAV 2712 & B & Foc VI & $0124 / 22$ & Rwanda & CAV 2862 & B & Foc VI & $0124 / 22$ & DRC \\
\hline CAV 2714 & B & Foc VI & $0124 / 22$ & Rwanda & CAV 2863 & $\mathrm{~B}$ & Foc VI & $0124 / 22$ & DRC \\
\hline CAV 2717 & B & Foc VI & $0124 / 22$ & Rwanda & CAV 2864 & B & Foc VI & 01212 & DRC \\
\hline CAV 2718 & B & Foc VI & $0124 / 22$ & Rwanda & CAV 2865 & B & Foc VI & 01212 & DRC \\
\hline CAV 2720 & B & Foc VI & 0125 & Rwanda & CAV 2866 & B & Foc VI & 01212 & DRC \\
\hline CAV 2721 & B & Foc VI & $0124 / 22$ & Rwanda & CAV 2867 & B & Foc VI & 01212 & DRC \\
\hline CAV 2722 & B & Foc VI & 0124 & Rwanda & CAV 2869 & B & Foc VI & 01222 & DRC \\
\hline CAV 2723 & A & A.P. & incomp. & Rwanda & CAV 2880 & $\mathrm{nt}$ & Foc VI (phylo) & incomp. & DRC \\
\hline CAV 2724 & B & Foc VI & $0124 / 22$ & Rwanda & CAV 2504 & B & Foc VI & $0124 / 22$ & Uganda \\
\hline CAV 2726 & B & Foc VI & 01222 & Rwanda & CAV 2505 & B & Foc VI & $0124 / 22$ & Uganda \\
\hline CAV 2459 & B & Foc VI & 01222 & Uganda & CAV 2510 & B & Foc VI & $0124 / 22$ & Uganda \\
\hline CAV 2461 & B & Foc VI & $0124 / 5$ & Uganda & CAV 2513 & B & Foc VI & 0128 & Uganda \\
\hline CAV 2464 & B & Foc VI & 0124 & Uganda & CAV 2522 & B & Foc VI & 0128 & Uganda \\
\hline CAV 2467 & B & Foc VI & $0124 / 22$ & Uganda & CAV 2523 & B & Foc VI & 0124 & Uganda \\
\hline CAV 2468 & B & Foc VI & $0124 / 22$ & Uganda & CAV 2528 & B & Foc VI & 01212 & Uganda \\
\hline CAV 2472 & B & Foc VI & 0124 & Uganda & CAV 2531 & B & Foc VI & $0124 / 22$ & Uganda \\
\hline CAV 2475 & B & Foc VI & 0124 & Uganda & CAV 2537 & B & Foc VI & $0124 / 22$ & Uganda \\
\hline CAV 2476 & B & Foc VI & $0124 / 22$ & Uganda & CAV 2539 & B & Foc VI & $0124 / 22$ & Uganda \\
\hline CAV 2478 & B & Foc VI & $0124 / 22$ & Uganda & CAV 2540 & B & Foc VI & $0124 / 22$ & Uganda \\
\hline CAV 2479 & B & Foc VI & $0124 / 22$ & Uganda & CAV 2541 & B & Foc VI & $0124 / 20 / 22$ & Uganda \\
\hline CAV 2480 & B & Foc VI & $0124 / 5$ & Uganda & CAV 2542 & B & Foc VI & 0124 & Uganda \\
\hline CAV 2481 & B & Foc VI & $0124 / 22$ & Uganda & CAV 2543 & B & Foc VI & 01222 & Uganda \\
\hline CAV 2482 & B & Foc VI & 0124 & Uganda & CAV 2544 & B & Foc VI & 01222 & Uganda \\
\hline CAV 2484 & B & Foc VI & 0124 & Uganda & CAV 2545 & B & Foc VI & 0124 & Uganda \\
\hline CAV 2485 & B & Foc VI & $0124 / 22$ & Uganda & CAV 2546 & B & Foc VI & $0124 / 22$ & Uganda \\
\hline CAV 2486 & B & Foc VI & 0124 & Uganda & CAV 2547 & B & Foc VI & $0124 / 22$ & Uganda \\
\hline CAV 2487 & B & Foc VI & 01222 & Uganda & CAV 2548 & B & Foc VI & 01222 & Uganda \\
\hline CAV 2489 & B & Foc VI & 0128 & Uganda & CAV 2549 & B & Foc VI & $0124 / 22$ & Uganda \\
\hline CAV 2490 & B & Foc VI & $0124 / 22$ & Uganda & CAV 2551 & B & Foc VI & $0124 / 5$ & Uganda \\
\hline CAV 2491 & B & Foc VI & $0124 / 5$ & Uganda & CAV 2552 & B & Foc VI & 0124 & Uganda \\
\hline CAV 2492 & B & Foc VI & 01212 & Uganda & CAV 2553 & B & Foc VI & $0124 / 5 / 22$ & Uganda \\
\hline CAV 2493 & B & Foc VI & 0124 & Uganda & CAV 2554 & B & Foc VI & 01222 & Uganda \\
\hline CAV 2494 & B & Foc VI & 01222 & Uganda & CAV 2556 & B & Foc VI & $0124 / 5$ & Uganda \\
\hline CAV 2495 & B & Foc VI & $0124 / 5$ & Uganda & CAV 2557 & B & Foc VI & $0124 / 22$ & Uganda \\
\hline CAV 2496 & B & Foc VI & 0128 & Uganda & CAV 2558 & B & Foc VI & 01222 & Uganda \\
\hline CAV 2497 & B & Foc VI & 0128 & Uganda & CAV 2559 & B & Foc VI & $0124 / 5$ & Uganda \\
\hline CAV 2498 & B & Foc VI & $0124 / 22$ & Uganda & & & & & \\
\hline CAV 2499 & B & Foc VI & 01212 & Uganda & & & & & \\
\hline CAV 2502 & B & Foc VI & 0124 & Uganda & & & & & \\
\hline
\end{tabular}


support of $100 \%$ (Fig. 3). These isolates were identified as $F$. sacchari (CAV 2804 and CAV 2837) and F. semitectum (CAV 2580 and CAV 2678), based on their TEF DNA sequences that were blasted against GenBank's nucleotide sequence repository.

Five isolates identified as $F$. oxysporum by morphological characters, including two from Rwanda and three from Tanzania, clustered loosely with the lineage VIII (VCG 01214) isolate CAV 189 (Fig. 3). Within this lineage, the pathogenic CAV 2734 isolate clustered with two nonpathogenic isolates (CAV 2581 and CAV 2723). When their TEF DNA sequences were blasted against the GenBank nucleotide sequence repository, isolates CAV 2723 and CAV 2734 from Rwanda shared a $99 \%$ identity with $F$. oxysporum f. sp. melonis. Isolate CAV 2581 from Tanzania shared a 100\% identity with a Grevillea robusta (A. Cunn. ex R. Br.) tree-associated $F$. oxysporum isolate and a human pathogenic $F$. oxysporum isolate, respectively, but with $99 \%$ query cover score.

Pathogenicity testing. Foc symptoms, ranging from yellow to dark brown discoloration of the rhizome, were caused by nine isolates (CAV 2576, CAV 2595, CAV 2599, CAV 2640, CAV 2734, CAV 2750, CAV 2764, CAV 2858, and CAV 2880) to Gros Michel plantlets. Of these, eight were identified as Foc lineage VI by phylogenetic analysis, and one $F$. oxysporum isolate loosely clustered with Foc lineage VIII VCG 01214 (CAV 2734).

\section{Discussion}

Foc in ECA proved to be more diverse than previously reported. VCGs 0128 and 01220 were found for the first time in Burundi,
DRC, Rwanda, Tanzania, and Uganda (Kangire et al. 2001; Ploetz et al. 1990; Rutherford 2001), while VCG 01222 is also first reported in Burundi, DRC, Rwanda, and Tanzania. VCG 01212 is reported in DRC and Rwanda for the first time. The occurrence of six VCGs in ECA, including VCG 01212 and a putative novel VCG, supports a hypothesis that Foc may have diversified in this region after being introduced from Asia (Bentley et al. 1998; Koenig et al. 1997). Such evolution may have occurred due to the selection of pathogenic variants imposed by the genetic distinctiveness of local cultivars. The putative new VCG was isolated from 'Sukari Ndiizi' (AAB), a variety that is relatively distinct from other Apple banana cultivars (Onyango 2007; Onyango et al. 2010). The ECA region has been recognized as the secondary banana diversity center after Southeast Asia, given the uniqueness of banana genotypes found in this region (Stover and Simmonds 1987).

Isolates in the VCG complex 0124/5/8/20/22, which is common in ECA, are among the most widespread worldwide (Bentley et al. 2001; Ploetz and Pegg 2000). A VCG complex constitutes a set of individual VCGs that, in some instances, pair with each other. VCG complex members are phylogenetically closely related, such as the VCGs in Foc lineage VI. The most common VCGs in ECA were members of the VCG complex 0124/22, which accounted for $70 \%$ of all Foc isolates collected. It is plausible that all the VCGs in lineage VI and complexes thereof are clonally related and evolved from one VCG by mutations in vic alleles, and that their distribution is related to the movement of banana planting materials and Focbanana coevolution (Bentley et al. 1998; Fourie et al. 2009). VCGs

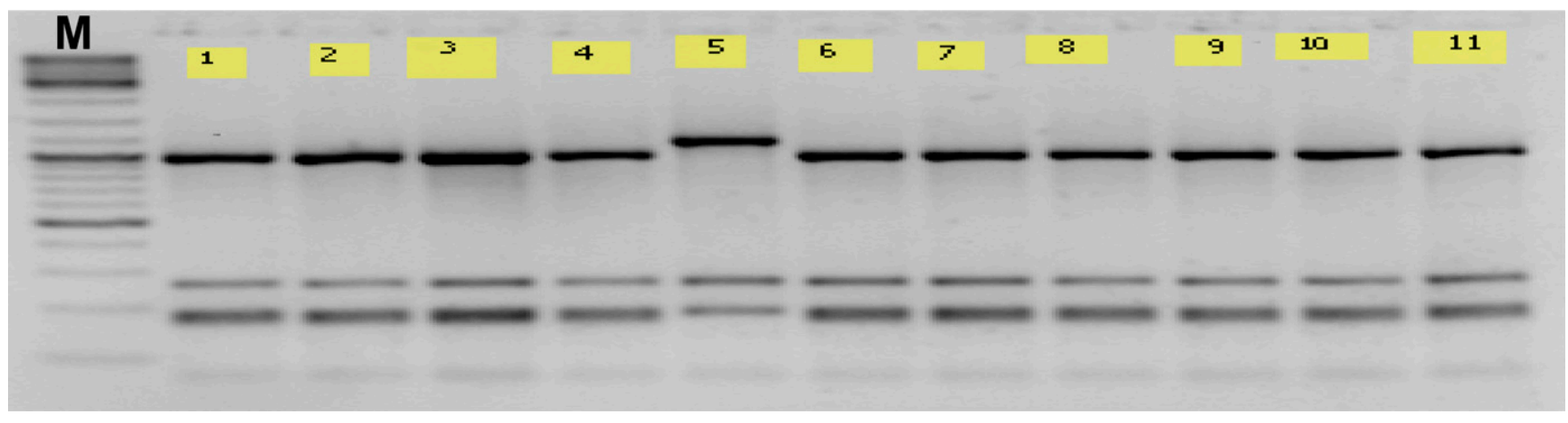

Fig. 2. Electrophoretic PCR-RFLP profiles of Bbvl-digested Fusarium oxysporum f. sp. cubense (Foc) isolates from Tanzania and Rwanda. $M=$ molecular weight marker. Lanes 1-4 and 6-11 represent lineage $\mathrm{VI}$, while lane 5 represents an Foc lineage VII member.

Table 2. Isolates of Fusarium oxysporum and other Fusarium species that were not compatible to known vegetative compatibility groups

\begin{tabular}{|c|c|c|c|c|c|}
\hline Isolate & Origin & GenBank accession no. & Morphology & Pathogenicity & Self-compatibility \\
\hline CAV2576 & Tanzania & KX365389 & F. oxysporum & + & $\mathrm{SC}^{\mathrm{a}}$ \\
\hline CAV2580 & Tanzania & KX365415 & $F$. semitecum & $n t^{\mathrm{b}}$ & $\mathrm{nt}$ \\
\hline CAV2581 & Tanzania & KX365392 & $F$. oxysporum & - & nt \\
\hline CAV2595 & Tanzania & KX365394 & F. oxysporum & + & $\mathrm{SC}$ \\
\hline CAV2599 & Tanzania & KX365395 & F. oxysporum & + & SC \\
\hline CAV2611 & Tanzania & KX365400 & F. oxysporum & - & $\mathrm{nt}$ \\
\hline CAV2633 & Tanzania & KX365403 & F. oxysporum & - & nt \\
\hline CAV2640 & Tanzania & KX365404 & F. oxysporum & + & SC \\
\hline CAV2678 & Rwanda & KX365416 & $F$. semitecum & nt & $\mathrm{nt}$ \\
\hline CAV2723 & Rwanda & KX365406 & F. oxysporum & - & $\mathrm{nt}$ \\
\hline CAV2734 & Rwanda & KX365407 & F. oxysporum & + & SC \\
\hline CAV2750 & Burundi & KX365408 & F. oxysporum & + & $\mathrm{HSI}^{\mathrm{c}}$ \\
\hline CAV2762 & Burundi & KX365409 & F. oxysporum & + & $\mathrm{SC}$ \\
\hline CAV2804 & Burundi & KX365417 & F. sacchari & nt & $\mathrm{nt}$ \\
\hline CAV2837 & DRC & KX365390 & F. sacchari & nt & nt \\
\hline CAV2858 & DRC & KX365412 & F. oxysporum & + & $\mathrm{SC}$ \\
\hline CAV 2880 & DRC & KX365414 & F. oxysporum & + & $\mathrm{SC}$ \\
\hline
\end{tabular}

${ }^{a} \mathrm{SC}$ : Self-compatible isolate.

${ }^{\mathrm{b}}$ nt: Non tested isolates, identified as non-Foc by microscopy and phylogenetic analysis, or by pathogenicity testing.

${ }^{\mathrm{c}}$ HSI: Heterokaryon self-incompatible. 
01212 and 01222 appear to be the more recent VCGs in lineage VI, as they are less widespread globally. A review of existing literature on Foc distribution worldwide indicates that VCG 01212 has only been reported in ECA, while VCG 01222 has been reported only in Southeast Asia and ECA (Bentley et al. 2001; Fourie et al. 2011; Ploetz and Pegg 2000).

The current study identified $13 F$. oxysporum isolates that did not pair with any of the known VCGs. Four of these were nonpathogens, while the remaining nine included eight that grouped with VCGs in Foc lineage VI, and one (CAV 2734) that grouped with nonpathogens and VCG 01214. It is thus most likely that isolate CAV 2734 could represent a putative novel VCG. The discovery of isolates that do not pair with known VCG testers is not unusual, and consistent with previous studies by Bentley et al. (1998). These authors reported five genotypes fitting the DNA fingerprinting group that included VCGs 0124, 0125, 0128, 01212, and 01220 (DFG IV), but which

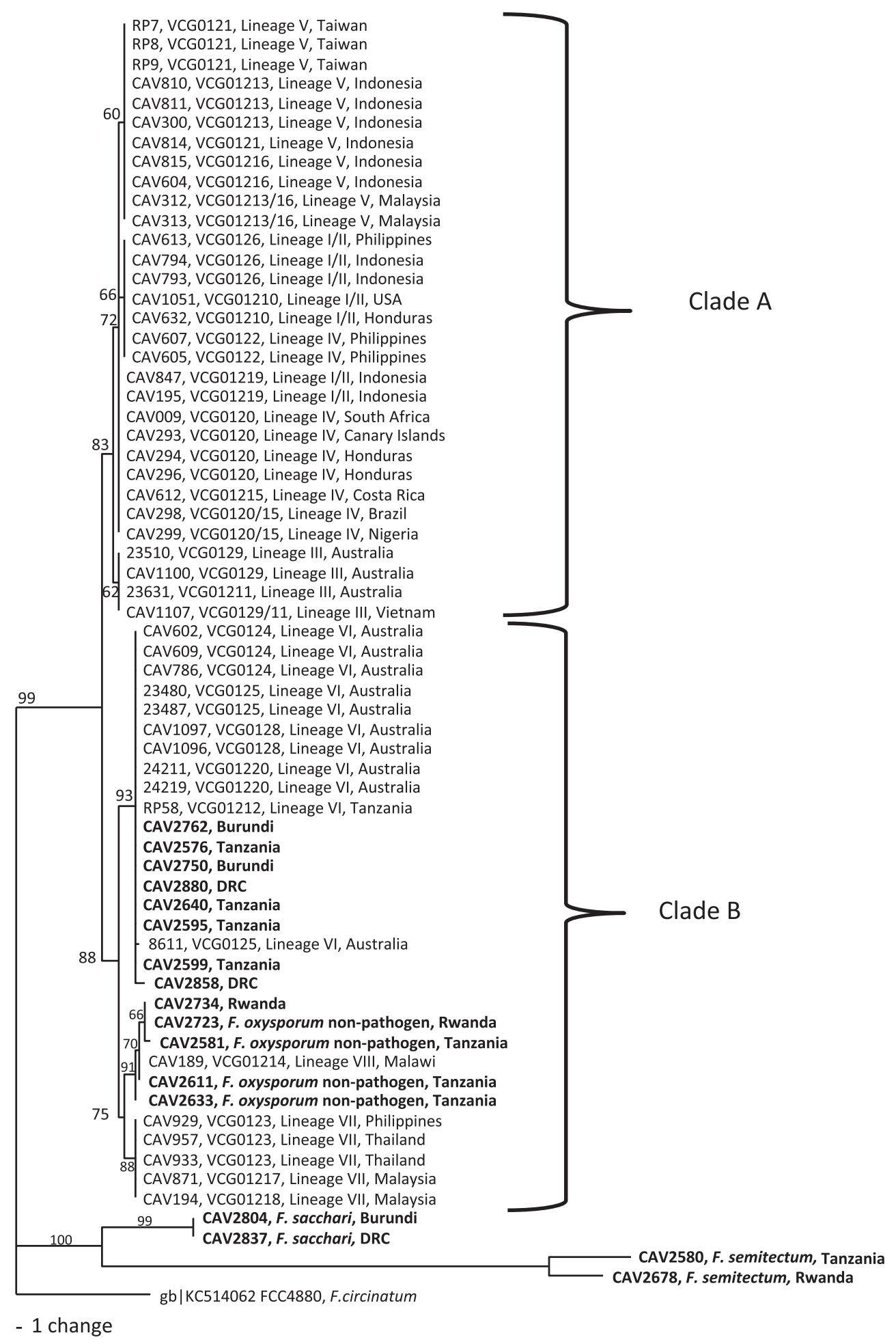

Fig. 3. Maximum likelihood phylogenetic tree of the translation elongation factor- $1 \alpha$ of Fusarium oxysporum $\mathrm{f}$. sp. cubense isolates from East and Central Africa. The isolates marked in bold were vegetatively incompatible with the 24 vegetative compatibility group tester strains. Bootstrap values greater than $(>60 \%)$ are indicated for maximum likelihood internodes where relevant. The tree is rooted with F. circinatum isolate FCC 4880. 
did not pair with any of them. It would thus be interesting to pair the unknown Foc strains found in this study with the genotypes described by Bentley et al. (1998). Also, it would be worthy of pairing unknown ECA strains with known VCG mutants generated from ECA isolates.

Fusarium isolates other than Foc have been isolated from banana in this study. These include $F$. semitecum (two isolates), $F$. sacchari (two isolates), and nonpathogenic $F$. oxysporum (four isolates). An association of nonpathogenic $F$. oxysporum strains and other Fusarium species with banana has been reported before (Belgrove et al. 2011; Nel et al. 2006; Zeng et al. 2013). F. semitecum can be associated with speckle disease, a blemish of banana peel that is not regarded as a significant constraint to non-export targeting production (Jones and Stover 2000), while $F$. sacchari has been associated with banana crown rot (Kamel et al. 2016). Nonpathogenic $F$. oxysporum isolates were shown to suppress Fusarium wilt development in the greenhouse, but not in the field (Belgrove et al. 2011).

The almost exclusive membership of Foc strains in ECA as lineage VI isolates has important disease management implications. This could allow for the development of molecular diagnostics for rapid detection, and the screening of cultivars for resistance to lineage VI. The formae specialis concept and physiological races in Foc, however, might mislead disease management strategies (Baayen et al. 2000; Fourie et al. 2009; Groenewald et al. 2006; Koenig et al. 1997; O'Donnell et al. 1998). Considerable genetic heterogeneity is found within Foc races 1 and 2, to the extent that no single strain can accurately represent Foc race 1 or 2 in experiments for the development of disease control technologies such as breeding for resistance and the development of diagnostic tools. The race structure in Foc therefore still needs to be clarified (Ploetz 2015), whereas virulence has been shown to be influenced by a number of variables including host age (Hart and Endo 1981) and method of inoculation (Kraft and Haglund 1978). Foc lineages may thus provide a classification system that is less laborious than VCG analyses, and more reliable than the race system, for pathogen detection and management.

In this study, VCG analysis, PCR-RFLPs of the ribosomal DNA's IGS region, phylogenetic analysis of the EF- $1 \alpha$ gene, and pathogenicity testing were used to investigate the genetic diversity of Foc in ECA. Three of the methods were mostly congruent in characterizing Foc, as isolates belonging to the same VCG were members of the same PCR-RFLP lineage and phylogenetic group. The exception was isolate CAV 2734, which loosely clustered with VCG 01214 (Foc lineage VIII), but did not fit the lineage VIII profile. VCG 01214 is genetically distinct from other Foc VCGs due to a potential African evolutionary origin (Bentley et al. 1998; Fourie et al. 2009; Koenig et al. 1997). More representative sampling and variety screening in the Burera district of northern Rwanda, where CAV 2734 was collected, could establish the prevalence and possible importance of this putative novel VCG.

\section{Acknowledgments}

We acknowledge the assistance of research workers at national agricultural research systems in ECA for collecting banana Fusarium wilt samples. These include Mr Sayi Bulili (Agricultural Research Institute, Maruku, Tanzania), Mr Mate Lukanda (University of Butembo, DRC), Mr Jules Ntamwira Bagula (Bioversity International CIALCA project, South Kivu, DRC) and Mr Léonidas Ntahimpera (Institut des Sciences Agronomiques du Burundi, Burundi). We thank Mrs. Anria Pretorius (Stellenbosch University, South Africa) for valuable technical assistance with isolation, single-sporing and maintenance of Foc isolates.

\section{Literature Cited}

Baayen, R. P., O’Donnell, K., Bonants, P. J. M., Cigelnik, E., Kroon, L. P. N., Roebroeck, J. A., and Waalwijk, C. 2000. Gene genealogies and AFLP analysis in the Fusarium oxysporum complex identify monophyletic and nonmonophyletic formae speciales causing wilt and rot disease. Phytopathology 90:891-900.

Belgrove, A., Steinberg, C., and Viljoen, A. 2011. Evaluation of nonpathogenic Fusarium oxysporum and Pseudomonas fluorescens for Panama disease control. Plant Dis. 95:951-959.

Bentley, S., Pegg, K. G., Moore, N. Y., Davis, R. D., and Buddenhagen, I. W. 1998. Genetic variation among vegetative compatibility groups of Fusarium oxysporum f. sp. cubense analysed by DNA fingerprinting. Phytopathology 88:1283-1293.
Bentley, S., Moore, N. Y., Pegg, K. G., Gerlach, K. S., and Smith, L. J. 2001. Genetic characterization and detection of Fusarium wilt. Pages 143-151 in: Banana Fusarium Wilt Management: Towards Sustainable Cultivation. A. B. Molina, N. H. Masdek, and K. W. Liew, eds. Proceedings of the International Workshop on the Banana Fusarium Wilt Disease, 18-20 October 1999, Genting Highlands Resort, Malaysia.

Butler, D. 2013. Fungus threatens top banana. Nature 504:195.

Dita, M., Waalwijk, C., Paiva, L. V., Souza, M. T., Jr., and Kema, G. H. J. 2011. A greenhouse bioassay for the Fusarium oxysporum f. sp. cubense $\mathrm{x}$ Grand Naine (Musa, AAA, Cavendish Subgroup) interaction. Acta Hortic. 897:377-380.

Edel, V., Steinberg, C., Avelange, I., Laguerre, G., and Alabouvette, C. 1995. Comparison of three molecular methods for the characterization of Fusarium oxysporum strains. Phytopathology 85:579-585.

Fourie, G., Steenkamp, E. T., Gordon, T. R., and Viljoen, A. 2009. Evolutionary relationships among the vegetative compatibility groups of Fusarium oxysporum f. sp. cubense. Appl. Environ. Microbiol. 75:4770-4781.

Fourie, G., Steenkamp, E. T., Ploetz, R. C., Gordon, T. R., and Viljoen, A. 2011. Current status of the taxonomic position of Fusarium oxysporum formae specialis cubense within the Fusarium oxysporum complex. Infect. Genet. Evol. 11:533-542.

Glass, N. L., Jacobson, D. J., and Shiu, P. K. T. 2000. The genetics of hyphal fusion and vegetative incompatibility in filamentous ascomycete fungi. Annu. Rev. Genet. 34:165-86.

Groenewald, S., Van Den Berg, N., Marasas, W. F. O., and Viljoen, A. 2006. The application of high-throughput AFLPs in assessing genetic diversity in Fusarium oxysporum f. sp. cubense. Mycol. Res. 110:297-305.

Guindon, S., Dufayard, J. F., Lefort, V., Anisimova, M., Hordijk, W., and Gascuel, O. 2010. New algorithms and methods to estimate maximumlikelihood phylogenies: assessing the performance of PhyML 3.0. Syst. Biol. 59:307-321.

Hart, L. P., and Endo, R. M. 1981. The effect of length of exposure to inoculum, plant age, root development and root wounding on Fusarium yellows of celery. Phytopathology 71:77-79.

Jones, D. R., and Stover, R. H. 2000. Fungal diseases of banana fruit: pre-harvest diseases. Pages 173-190 in: Diseases of Banana, Abaca and Enset. D. R. Jones, ed. CABI Publishing, New York.

Kamel, M. A. M., Cortesi, P., and Saracchi, M. 2016. Etiological agents of crown rot of organic bananas in Dominican Republic. Postharvest Biol. Technol. 120: 112-120.

Kangire, A., Rutherford, M. A., and Gold, C. S. 2001. Distribution of fusarium wilt and the populations of Fusarium oxysporum $\mathrm{f}$. sp. cubense on bananas in Uganda. Pages 152-161 in: Banana Fusarium Wilt Management: Towards Sustainable Cultivation. A. B. Molina, N. H. Masdek, and K. W. Liew, eds). Proceedings of the International Workshop on the Banana Fusarium Wilt Disease, 18-20 October 1999, Genting Highlands Resort, Malaysia.

Karamura, E. B., Frison, E. A., Karamura, D. A., and Sharrock, S. 1999. Pages 401-412 in: Banana production systems in eastern and southern Africa Bananas and Food Security. INIBAP, Montpellier, France.

Karangwa, P., Blomme, G., Beed, F., Niyongere, C., and Viljoen, A. 2016. The distribution and incidence of banana fusarium wilt in subsistence farming systems in East and Central Africa. Crop Prot. 84:132-140.

Katoh, K., Kuma, K. I., Toh, H., and Miyata, T. 2005. MAFFT version 5: improvement in accuracy of multiple sequence alignment. Nucleic Acids Res. 33:511-518.

Koenig, R. L., Ploetz, R. C., and Kistler, H. C. 1997. Fusarium oxysporum f. sp. cubense consists of a small number of divergent and globally distributed clonal lineages. Phytopathology 87:915-923.

Kraft, J. M., and Haglund, W. A. 1978. A reappraisal of the race classification of Fusarium oxysporum $\mathrm{f}$. sp. pisi. Phytopathology 68:273-275.

Kung'u, J. N., and Jeffries, P. 1998. Vegetative compatibility groups within Kenyan populations of Fusarium oxysporum f. sp. cubense. Acta Hortic. 490: 305-313.

Lefort, V., Longueville, J.-E., and Olivier Gascuel, O. 2017. SMS: Smart Model Selection in PhyML. Mol. Biol. Evol. 34:2422-2424.

Leslie, J. F., and Summerell, B. A. 2006. The Fusarium Laboratory Manual Blackwell Publishing-Wiley, Hoboken, NJ.

Lynam, J. K. 2000. Market development and production potential for banana and plantain. Acta Hortic. 540:39-53.

Nel, B., Steinberg, C., Labuschagne, N., and Viljoen, A. 2006. Isolation and characterization of non-pathogenic Fusarium oxysporum isolates from the rhizosphere of healthy banana plants. Plant Pathol. 55:207-216.

O’Donnell, K., Kistler, H. C., Cigelnik, E., and Ploetz, R. C. 1998. Multiple evolutionary origins of the fungus causing Panama disease of banana: concordant evidence from the nuclear and mitochondrial gene genealogies. Proc. Natl. Acad. Sci. USA 95:2044-2049.

Onyango, M. A. 2007. Characterization of East African Accessions of Musa AAB 'Apple' and Musa AA 'Muraru' Dessert Bananas. PhD dissertation, University of Hawaii, Manoa.

Onyango, M., Haymer, D., Keeley, S., and Manshardt, R. 2010. Analysis of genetic diversity and relationships in East African 'Apple banana' (AAB genome) and 'Muraru' (AA genome) dessert bananas using microsatellite markers. Acta Hortic. 879:623-636. 
Ploetz, R. C., and Correll, J. C. 1988. Vegetative compatibility among races of Fusarium oxysporum f.sp. cubense. Plant Dis. 72:325-328.

Ploetz, R. C., and Pegg, K. G. 2000. Fusarium wilt. Pages 143-159 in: Diseases of Banana, Abaca and Enset. D. R. Jones, ed. CABI Publishing, New York.

Ploetz, R. C., Herbert, J., Sebasigari, K., Hernandez, J. H., Pegg, K. G., Ventura, J. A., and Mayato, L. S. 1990. Importance of Fusarium wilt in different banana growing regions. Pages 9-26 in: Fusarium Wilt of Banana. R. C. Ploetz, ed. APS Press, St Paul, MN.

Ploetz, R. 2015. Management of Fusarium wilt of banana: A review with special reference to tropical race 4. Crop Prot. 73:7-15.

Rutherford, M. A. 2001. Fusarium wilt of banana in East Africa. Pages 86-94 in: Banana Fusarium Wilt Management: Towards Sustainable Cultivation. A. B.

Molina, N. H. Masdek, and K. W. Liew, eds. Proceedings of the International

Workshop on the Banana Fusarium Wilt Disease, 18-20 October 1999, Genting Highlands Resort, Malaysia.

Simmonds, N. W. 1966. Bananas, 2nd Ed. Longmans, London.
Stover, R. H. 1962. Fusarial wilt (Panama disease) of bananas and other Musa species. Pages 167-188 in: Banana, Plantain and Abaca Diseases. Commonwealth Mycological Institute, Kew, Surrey, UK

Stover, R. H., and Buddenhagen, I. W. 1986. Banana breeding: polyploidy, disease resistance and productivity. Fruits 41:175-191.

Stover, R. H., and Simmonds, N. W. 1987. Bananas, 3rd Ed. Longman, London.

Stover, B. C., and Müller, K. F. 2010. TreeGraph 2: Combining and visualizing evidence from different phylogenetic analyses. BMC Bioinformatics 11:7.

Tavare, S. 1986. Some probabilistic and statistical problems in the analysis of DNA sequences. Lect. Math Life Sci. 17:57-86.

Waite, B. H., and Stover, R. H. 1960. Studies on Fusarium wilt of bananas: VI Variability and the cultivar concept in Fusarium oxysporum f. sp. cubense. Can. J. Bot. 38:985-994.

Zeng, L-S., Zhao, Z-H., Lu, S., Xi, Z.-J., Li, M.-H., Xi, P.-G., and Jiang, Z.-D. 2013. The Fusarium species isolated from banana and their phylogenetic relationships. Mycosystema 32:617-632. 